\title{
Meshless Methods and Partition of Unity Finite Elements
}

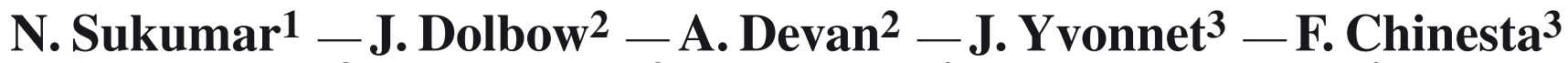 D. Ryckelynck ${ }^{3}-$ P. Lorong $^{3}-$ I. Alfaro ${ }^{4}-$ M. A. Martínez ${ }^{4}$ E. Cueto ${ }^{4}-$ M. Doblaré 4}

${ }^{1}$ Department of Civil and Environmental Engineering, University of California, One Shields Avenue, Davis, CA 95616. U.S.A.

nsukumar@ucdavis.edu

2 Department of Civil and Environmental Engineering, Duke University Box 90287, Durham, NC 27708-0287. U.S.A.

jdolbow@duke.edu

3 LMSP (Laboratoire de Mécanique des Systèmes et des Procédés) UMR 8106 CNRS-ENSAM-ESEM

151 boulevard de l'Hôpital, F-75013 Paris, France

julien.yvonnet@paris.ensam.fr

${ }^{4}$ Aragón Institute of Engineering Research. University of Zaragoza. Edificio Betancourt. María de Luna, 5, E-50018. Zaragoza, Spain ecueto@unizar.es

ABSTRACT. This paper encompasses the main conclusions obtained in the mini-symposium New and Advanced Numerical Strategies in Forming Processes Simulation, held during the 6th International ESAFORM Conference on Material Forming (Salerno 2003), particularly those aspects dealing with meshless and partition of unity methods applied to the simulation of forming processes.

KEYWORDS: Meshless methods, Partition of Unity methods, Natural Neighbour Galerkin methods, Forming Processes. 


\section{Introduction}

With an aim towards alleviating the need for mesh re-generation in moving boundary (such as crack growth) and large deformation problems, there has been significant interest in the development and application of meshless (or meshfree) methods. The impetus in this direction emanated from the work by Nayroles and co-workers who proposed the diffuse element method (DEM) (Nayroles et al., 1992) in 1992, and since then there have been many new developments to this class of Galerkin methods. A detailed discussion and comparison of different meshless and particle methods can be found in references (Belytschko et al., 1998); ( $\mathrm{Li}$ and Liu, 2002). The meshless paradigm has provided new insights into the finite element method (Babuška and Melenk, 1996); (Duarte and Oden, 1996), and also brought out the intimate link between scattered data approximation, computational geometry, and the numerical solution of PDEs. In particular, the partition of unity framework (Babuška and Melenk, 1996) is a powerful technique to model discontinuities and singularities through local enrichment within a finite element setting. Level set and fast marching methods (FMM) (Sethian, 1999) are well-known interface-capturing techniques in which the interface is represented as the zero level contour of a function (level set) of one higherdimension. The coupling of partition of unity techniques to level set methods is an appealing means to carry out geometric computations, evaluate enrichment functions (especially in 3-d), and to evolve interfaces on a fixed finite element mesh.

The next sections deal with the description and analysis of some of the most popular meshless and partition of unity methods. In section 2 we review the methods based on moving leasts squares approximation. On section 3 we discuss methods based on natural neighbour interpolation, the so-called natural neighbour Galerkin or natural element methods (Sukumar et al., 1998) (Cueto et al., 2000) (Cueto et al., 2003b). Different approaches to enforce essential boundary conditions in these methods exist. In section 3.1 we describe the approach followed by (Cueto et al., 2000) (Cueto et al., 2002), based on the use of $\alpha$-shapes. In section 3.2 we review the use of the visibility criteria, as in (Yvonnet et al., 2004). Finally, in section 4, we analyse some applications of the Partition of Unity paradigm to add discontinuities to the essential (usually the displacement) field. This is the basis of the so-called extended finite element methods (X-FEM) (Moës et al., 1999) (Dolbow, 1999) (Sukumar et al., 2001) or partition-of-unity finite element methods (PUFEM) (Melenk and Babuška, 1996).

\section{Methods based on moving least squares approximation}

Given a set of scattered nodes in $\mathbb{R}^{d}(d=1-3)$ with prescribed nodal data, a surface approximation can be constructed without the need for any (finite element) a priori connectedness information between the nodes. This viewpoint is adopted in meshless Galerkin methods, where well-known methods from data approximation theory (Lancaster and Salkauskas, 1981) (Sibson, 1980) are used to construct the trial and test spaces. We first touch upon moving least squares (MLS) approximants (Lancaster and Salkauskas, 1981) that are used in the Element-Free Galerkin (EFG) method as well 
as in many of the other meshless methods (Li and Liu, 2002), and then discuss natural neighbor-based interpolant schemes. In the MLS approximation, the trial function $u^{h}$ for a scalar-valued function $u$ is written as (Belytschko et al., 1998)

$$
u^{h}(\mathbf{x})=\sum_{j=1}^{m} p_{j}(\mathbf{x}) a_{j}(\mathbf{x}) \equiv \mathbf{p}^{T}(\mathbf{x}) \mathbf{a}(\mathbf{x}),
$$

where $m$ is the number of terms in the basis function vector $\mathbf{p}$, and $a_{j}$ are coefficients which are found by minimizing the quadratic functional $J$ :

$$
J(\mathbf{x})=\sum_{I=1}^{n} w_{I}(\mathbf{x})\left[\mathbf{p}^{T}\left(\mathbf{x}_{I}\right) \mathbf{a}(\mathbf{x})-u_{I}\right]^{2},
$$

where $w_{I}(\mathbf{x}) \equiv w\left(\mathbf{x}-\mathbf{x}_{I}\right) \geq 0$ is a weight function with compact support. On taking the extremum of $J$ and after some simplification, we obtain

$$
u^{h}(\mathbf{x})=\sum_{I=1}^{n} \phi_{I}(\mathbf{x}) u_{I},
$$

where $u_{I}$ are nodal parameters and the EFG shape functions are given by

$$
\phi_{I}(\mathbf{x})=\sum_{j=1}^{m} p_{j}(\mathbf{x})\left[\mathbf{A}^{-1}(\mathbf{x}) \mathbf{B}(\mathbf{x})\right]_{j I} .
$$

Since $\phi_{I}\left(\mathbf{x}_{J}\right) \neq \delta_{I J}$, the shape functions do not interpolate nodal data. Moreover, shape functions on the interior of the domain do not necessarily vanish on the boundary, complicating the imposition of essential boundary condition in a Galerkin method (Wagner and Liu, 2001). In the above equation, the matrices $\mathbf{A}$ (moment matrix) and $\mathbf{B}$ are given by

$$
\begin{aligned}
& \mathbf{A}(\mathbf{x})=\sum_{I=1}^{n} w_{I}(\mathbf{x}) \mathbf{p}\left(\mathbf{x}_{I}\right) \mathbf{p}^{T}\left(\mathbf{x}_{I}\right) \\
& \mathbf{B}(\mathbf{x})=\left[w_{1}(\mathbf{x}) \mathbf{p}\left(\mathbf{x}_{1}\right), \ldots, w_{n}(\mathbf{x}) \mathbf{p}\left(\mathbf{x}_{n}\right)\right] .
\end{aligned}
$$

For smooth basis functions, the shape functions inherit the continuity of the weight function. This property provides a simple means to construct $C^{k}(k \geq 0)$ trial and test approximations.

In the EFG method, each node is associated with a domain of influence, which is the support of the weight function $w_{I}$, with $w_{I}(\mathbf{x})>0$ in its interior and $w_{I}(\mathbf{x})=0$ outside it. Typically, domains of influence are circular or rectangular in 2-d, and Gaussian or polynomial (spline) weight functions are used (Belytschko et al., 1998). The approximant used in the reproducing kernel particle method also bears close affinity to the MLS-scheme (Li and Liu, 2002). 


\section{Natural neighbour Galerkin methods}

The notion of natural neighbours introduced by Sibson (Sibson, 1981) is an attractive alternative to MLS approximants. The Sibson (Sibson, 1981) and the Laplace (Christ et al., 1982) interpolants are both based on natural neighbors. The definition of natural neighbors relies on the Voronoi diagram of a nodal set. For ease of exposition, we restrict our attention to two-dimensions. The Voronoi diagram partitions a set of nodes into regions such that any point within the (first-order) Voronoi cell $\mathcal{V}\left(n_{I}\right)$ is closer to node $n_{I}$ than to any other node. In Figure 1, the Voronoi diagram for a set of seven nodes is shown. A point $p$ is introduced into the domain $\Omega$. Now, the Voronoi diagram for $p$ along with the seven nodes is constructed. If $p$ and node $n_{I}$ have a common Voronoi facet, then node $n_{I}$ is said to be a natural neighbor of the point $p$ (Sibson, 1981). In Figure 1, the point $p$ has five natural neighbors (filled circles).

The Sibson shape function of $p$ with respect to a natural neighbor $I$ is defined as the ratio of the area of the second-order Voronoi cell $\left(A_{I}\right)$ to the total area $A$ of the Voronoi cell of $p$ :

$$
\phi_{I}(\mathbf{x})=\frac{A_{I}(\mathbf{x})}{A(\mathbf{x})}, \quad A(\mathbf{x})=\sum_{J=1}^{n} A_{J}(\mathbf{x}),
$$

where $n=5$ and $A$ is the polygonal (dotted line) area associated with $p$ (Figure 1). Let $s_{I}$ be the length of the Voronoi facet, and $h_{I}=d\left(\mathbf{x}, \mathbf{x}_{I}\right)$ the distance between $p$ and node $I$. The Laplace shape function for node $I$ is defined as (Christ et al., 1982):

$$
\phi_{I}(\mathbf{x})=\frac{\alpha_{I}(\mathbf{x})}{\sum_{J=1}^{n} \alpha_{J}(\mathbf{x})}, \quad \alpha_{J}(\mathbf{x})=\frac{s_{J}(\mathbf{x})}{h_{J}(\mathbf{x})} .
$$

The Sibson and Laplace shape functions are non-negative $\left(\phi_{I} \geq 0\right)$, interpolate nodal data, and can exactly reproduce a linear field (linearly complete) (Sukumar, 1998). As opposed to MLS approximants, the construction of these shape functions is purely geometric with no user-defined (such as the weight function $w$ or its support size) parameters involved in its definition, and a robust approximation is realized for non-uniform nodal discretizations in multi-dimensions. The support of shape functions based on natural neighbor and MLS-schemes is shown in Figure 2. Consider the discrete weak form for the Laplace equation: $\int_{\Omega} \nabla u^{h} \cdot \nabla\left(\delta u^{h}\right) d \Omega=0$. On noting the support of the meshless shape functions illustrated in Figure 2, we can infer that accurate numerical integration of the weak form is an issue in meshless methods, since the intersection of shape function supports do not coincide with the integration (triangulation or quadrangulation) cells. In (Cueto et al., 2003b), an overview of natural neighbor-based Galerkin methods with applications in solid and fluid mechanics is presented.

It has been demonstrated (see (Sukumar et al., 1998) and references therein) that the Sibson approximation is strictly an interpolant along the boundary of the convex 


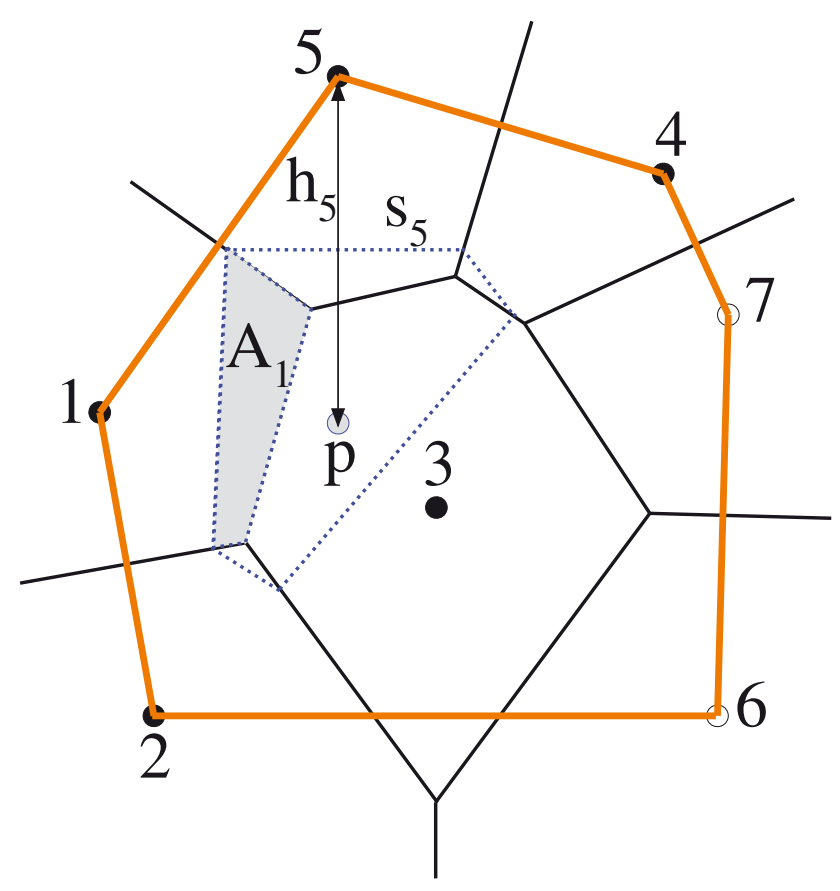

Figure 1. Sibson and Laplace shape functions
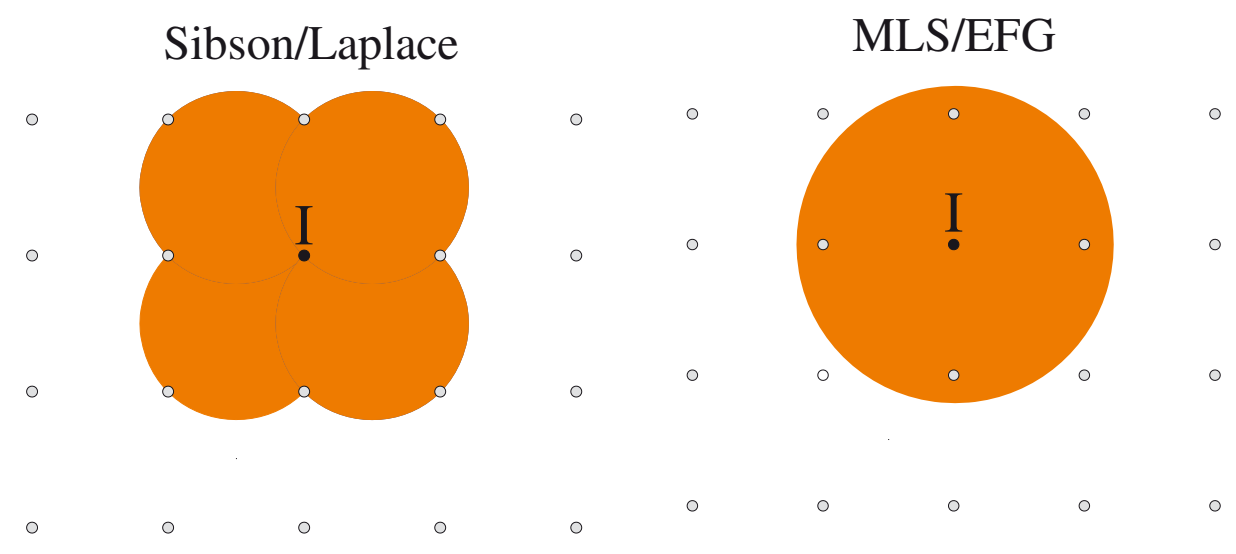

Figure 2. Support of meshless shape functions. The MLS/EFG shape function on the right is often scaled in size to cover many more nodes at each location

hull of the cloud of points. Thus, for non-convex domains, a suitable method to enforce essential boundary conditions is necessary. Among different possibilities, two main methods have emerged. The first is based on the use of $\alpha$-shapes (Edelsbrunner and Mücke, 1994), that allows both to impose linear essential boundary conditions exactly in a straightforward manner and to build models from clouds of points only without the need of any definition of the boundary. The second method is based on the use of constrained Voronoi diagrams (Yvonnet et al., 2004). Both approaches are indeed closely related. In the following sections they are reviewed. 


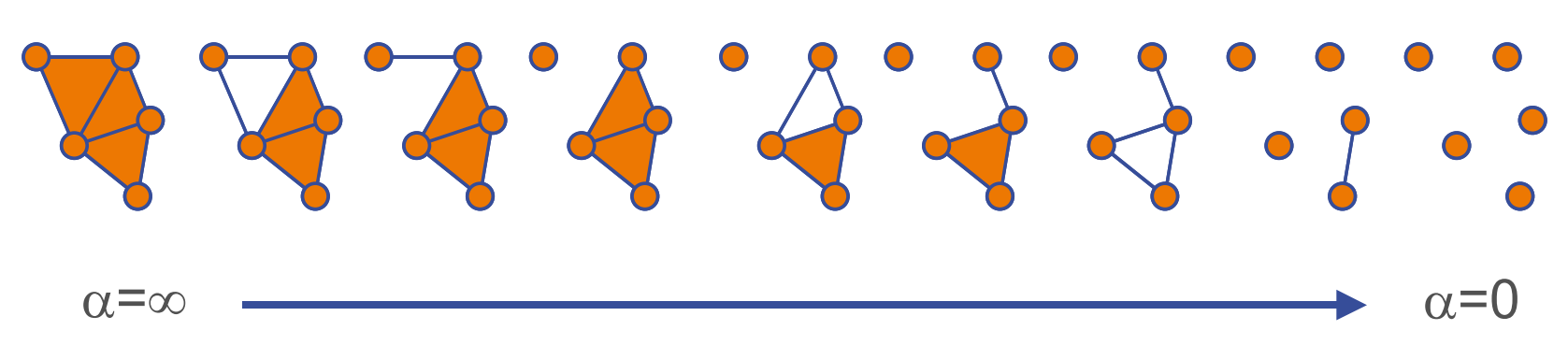

Figure 3. Some members of the family of shapes for a cloud of five points

\subsection{The $\alpha$-shapes based Natural Element Method ( $\alpha$-NEM)}

A cloud of points itself (without any connectivity between them, nor explicit definition of the boundary) defines a finite number of shapes. For a proper definition of the concept of shape, Edelsbrunner (Edelsbrunner et al., 1983) established the definition of a complete family of shapes of a cloud of points, based on the Delaunay triangulation (tetrahedralization in $\mathbb{R}^{3}$ ), that is unique for a given cloud.

In essence, an $\alpha$-shape is a polytope that is not necessarily convex nor connected. It is triangulated by a subset of the Delaunay triangulation of the nodes, and hence the empty circumcircle criterion holds. Let $N$ be a finite set of points in $\mathbb{R}^{3}$ and $\alpha$ a real number, with $0 \leq \alpha<\infty$. A $k$-simplex $\sigma_{T}$ with $0 \leq k \leq 3$ is defined as the convex hull of a subset $T \subseteq N$ of size $|T|=k+1$. Let $b$ be an $\alpha$-ball, i.e., an open ball of radius $\alpha$. A $k$-simplex $\sigma_{T}$ is said to be $\alpha$-exposed if there exist an empty $\alpha$-ball $b$ with $T=\partial b \bigcap N$ where $\partial \Omega$ refers to the boundary of the ball. In other words, a $k$-simplex is said to be $\alpha$-exposed if an $\alpha$-ball that passes through its defining points contains no other point of the set $N$.

We can now define the family of sets $F_{k, \alpha}$ as the sets of $\alpha$-exposed $k$-simplexes for the given set $N$. This allows us to define an $\alpha$-shape of the set $N$ as the polytope whose boundary consists of the triangles in $F_{2, \alpha}$, the edges in $F_{1, \alpha}$ and the vertices or nodes in $F_{0, \alpha}$. As remarked before, an $\alpha$-shape is a polytope that can be triangulated by a subset of the Delaunay triangulation or tetrahedralization, i.e., by an $\alpha$-complex. An example of two-dimensional family of $\alpha$-shapes for a simple cloud of five points is shown in figure 3 .

This definition of the shape of a cloud of points allows us to dynamically extract the shape of the cloud of points as it evolves during the process of deformation. If there exists a proper relationship between the $\alpha$ value and the nodal distance, $h$, - in the sense that $\alpha$ is a measure of the level of detail up to which the domain is represented, and must be of the order of $h-$ a proper conservation of the mass of the problem is achieved (Cueto et al., 2003b).

In addition, it can be proved that a proper imposition of essential boundary conditions is achieved if the neighbourhood between nodes is restricted to those pertaining to the same simplex in a certain $\alpha$-complex (Cueto et al., 2000) (Cueto et al., 2003a). 
As an example of the capabilities of the method, a simulation of the forging process of a tool is presented in the next section.

\section{Example: Forging of a workpiece}

In this example we consider the forging process of a workpiece, simulated assuming plane strain. The governing equations are:

1) Equilibrium equations (balance of linear momentum in the absence of inertial and body forces):

$$
\nabla \cdot \sigma=\mathbf{0} .
$$

2) Material incompressibility:

$$
\nabla \cdot \mathbf{v}=0 .
$$

The material behaviour is supposed to be governed by a Norton-Hoff-like law, i.e.,

$$
\boldsymbol{\sigma}=-p \mathbf{I}+2 \mu(\mathbf{D}) \mathbf{D},
$$

where the viscosity is a function of the second invariant of the strain rate tensor, $\mathbf{D}$, namely

$$
\mu(\mathbf{D})=\mu_{0}(\sqrt{2 \mathbf{D}: \mathbf{D}})^{n-1}
$$

where $\mu_{0}$ the so-called consistency coefficient and $n$ the pseudo-plasticity coefficient. In the numerical example we have considered $\mu_{0}=1.0$ and $n=0.3$.

If we write the incremental variational equations about time $t$ we arrive to:

$$
\int_{\Omega(t+\Delta t)}\left(-\left(p^{t}+\Delta p\right) \mathbf{I}+2 \mu\left(\mathbf{D}^{t}+\Delta \mathbf{D}\right)\right): \mathbf{D}^{*} d \Omega=0
$$

Due to the non-linear character of the constitutive equations, an iterative approach has been employed, using the Newton-Raphson scheme, thus leading to

$$
\begin{aligned}
& \int_{\Omega(t+\Delta t)}\left(-\Delta \Delta p \mathbf{I}+2 \mu\left(\frac{\partial \mu\left(\mathbf{D}_{k}^{t+\Delta t}\right)}{\partial \mathbf{D}}: \Delta \Delta \mathbf{D}\right) \mathbf{D}_{k}^{t+\Delta t}+\right. \\
& \left.+2 \mu\left(\mathbf{D}_{k}^{t+\Delta t}\right) \Delta \Delta \mathbf{D}\right): \mathbf{D}^{*} d \Omega= \\
& =-\int_{\Omega(t+\Delta t)}\left(-p_{k}^{t+\Delta t} \mathbf{I}+2 \mu\left(\mathbf{D}_{k}^{t+\Delta t}\right) \mathbf{D}_{k}^{t+\Delta t}\right): \mathbf{D}^{*} d \Omega
\end{aligned}
$$

The incremental form of the incompressibility condition results

$$
\int_{\Omega(t+\Delta t)} \nabla \cdot(\Delta \Delta \mathbf{v}) p^{*} d \Omega=-\int_{\Omega(t+\Delta t)} \nabla \cdot\left(\mathbf{v}_{k}^{t+\Delta t}\right) p^{*} d \Omega
$$




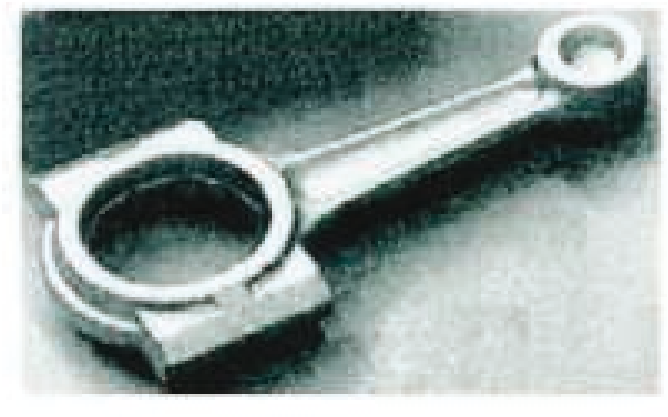

(a)

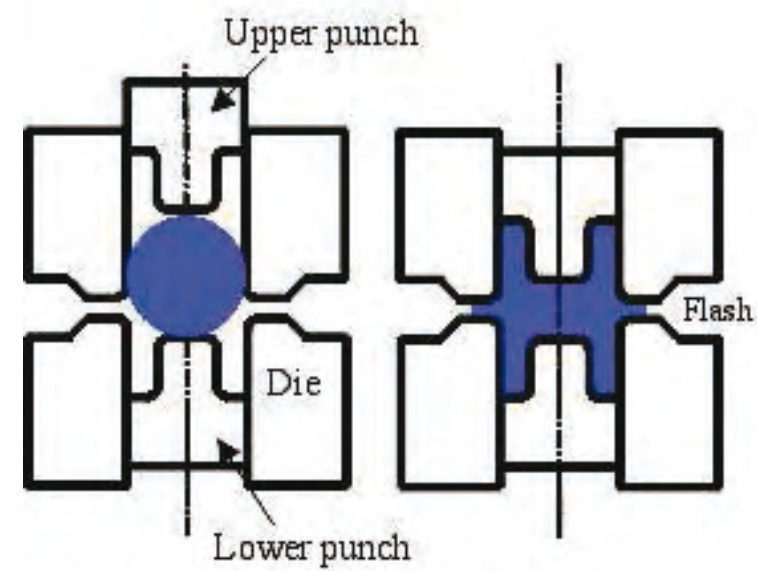

(b)

Figure 4. The piece to be forged (a) and the geometry of the simulated cross-section (b)

We took a mixed $\mathcal{C}^{0}-\mathcal{C}^{-1}$ natural neighbour approximation in velocities-pressures, as in (Cueto et al., 2003b), which does not exhibit spurious modes, although it does not satisfy the LBB condition.

The geometry of the piece is shown in Figure 4(a). The simulation deals with the forging of the central region of the piece, justifying the assumption of plane strain (see Figure 4(b)).

The equivalent plastic strain at time steps 1, 100, 150 and 173 are shown in Figure 5. Very accurate results were obtained in spite of the large strains and displacements involved in the simulation.

\subsection{The Constrained Natural Element Method (C-NEM)}

The fundamental basis of the C-NEM is the introduction of a visibility criterion to restrict influent nodes (natural neighbours). This criterion is necessary to avoid the parasitic influences between nodes over non convex boundaries. It must interact with a description of the boundary in a CAD sense. Such a criterion has been used in other meshless methods where similar problems are found (Organ et al., 1996). In this approach, a point $\mathbf{x}$ is in the domain of influence of a node $\mathbf{n}_{i}$ if $\mathbf{x}$ lies within the region where the shape function is non-zero and it is visible from node $\mathbf{n}_{i}$ when domain boundaries are assumed opaque (Organ et al., 1996). Remarkably, it has been shown in (Yvonnet et al., 2004) that the application of the visibility criterion in the context of NEM doesn't suffer from spurious discontinuities encountered in EFG method or RKPM (Organ et al., 1996). This property is due to the variable shape of the support with respect to the surrounding nodes (natural neighbours). Hence no additional criteria are necessary (diffraction, transparency) (Organ et al., 1996). Then its main 


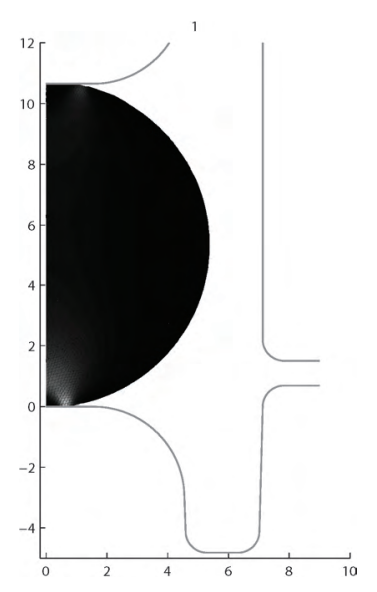

(a) 1st time step

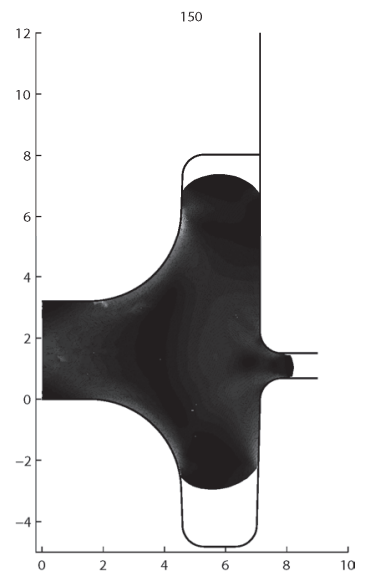

(c) 150th time step
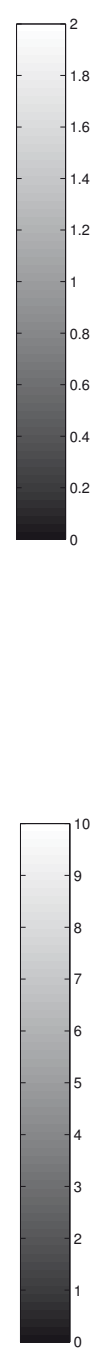

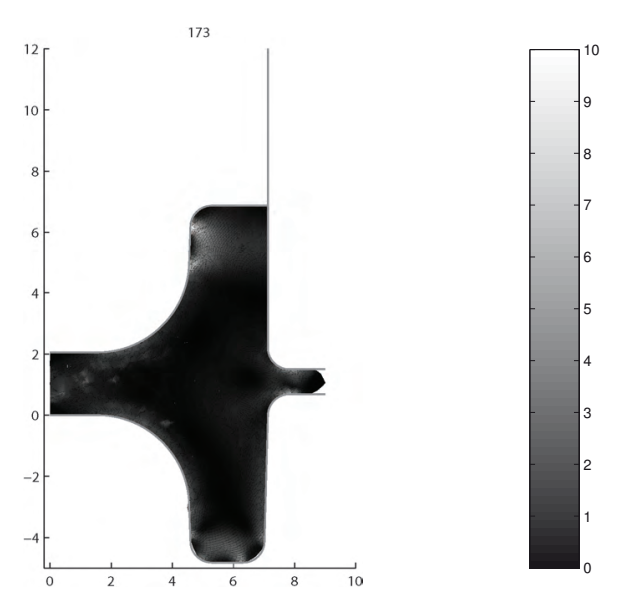

(d) 173th time step

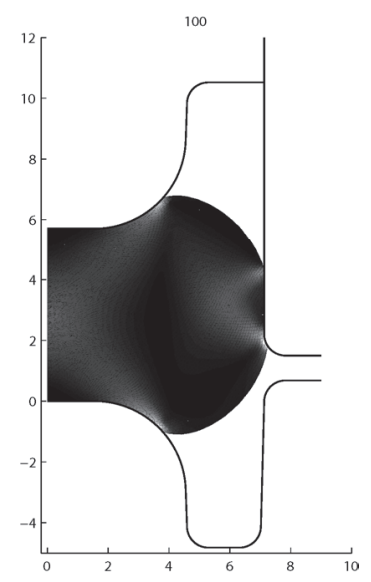

(b) 100th time step

Figure 5. Equivalent plastic strain at different time steps of the process

advantages are: no additional computational efforts, and less difficulties to extend the method for 3D analysis.

We introduce a modified, coined as 'constrained' Voronoi diagram for non convex domains. We can view the constrained Voronoi diagram (CVD) or bounded Voronoi diagram as a strict dual to the constrained Delaunay tessellation. The CVD is defined as follows: the intersection of the CVD with the domain closure is composed with cells $T_{i}^{C}$, one for each node $\mathbf{n}_{i}$, such that any given point $\mathbf{x}$ in $T_{i}^{C}$ is closer to $\mathbf{n}_{i}$ than any other node $\mathbf{n}_{j}$ visible from point $\mathbf{x}$.

$$
T_{i}^{C}=\left\{\mathbf{x} \in \mathbb{R}^{n}: d\left(\mathbf{x}, \mathbf{n}_{i}\right)<d\left(\mathbf{x}, \mathbf{n}_{j}\right), \forall j \neq i, \mathbf{n}_{j} \text { visible from } \mathbf{n}_{i}\right\}
$$

The CVD is deduced from the constrained Delaunay tessellation. We introduced the CVD for the two following reasons: (a) once such diagram is constructed, classical 
algorithms for the computation of the shape functions (Braun and Sambridge, 1995) can be applied directly because connections between natural neighbours are removed by the visibility criterion over any non convex domain, and (b) the constrained Voronoi cells match precisely integration domains that are used in our integration scheme.

We now define the constrained natural neighbours (C-n-n) like they are first selected by the classical natural neighbours criteria (Braun and Sambridge, 1995) (Sukumar et al., 1998) (lying on an empty sphere, sharing one Voronoi cell), and are then restricted by the visibility criterion. The trial and test functions result :

$$
\mathbf{u}^{h}(\mathbf{x})=\sum_{i=1}^{V} \phi_{i}^{C}(\mathbf{x}) \mathbf{u}_{i}
$$

where $\mathbf{u}_{i}$ are the nodal unknowns, $V$ the number of neighbours nodes visible from point $\mathrm{x}$ and $\phi_{i}^{C}$ the constrained natural neighbours shape functions. The C-n-n shape functions are similar to NEM shape functions but the support can be different (Yvonnet et al., 2004) and its computation is done on the basis of the CVD.

In Figure 6 we can see that for a point $\mathrm{x}$ introduced in the constrained Voronoi diagram, only nodal values from $\mathbf{n}_{1}, \mathbf{n}_{2}$ and $\mathbf{n}_{3}$ will contribute to the interpolation, because $\mathbf{n}_{4}$ does not share a constrained Voronoi cell with $\mathbf{x}$. We can conclude that: (a) influences from nodes lying over non convex domains vanish near non convex boundaries because the constrained voronoi diagram removes connexions between non mutually visible natural neighbours, and (b) linear interpolation (Sukumar et al., 1998) is satisfied on any boundary (convex or not) by the same considerations.

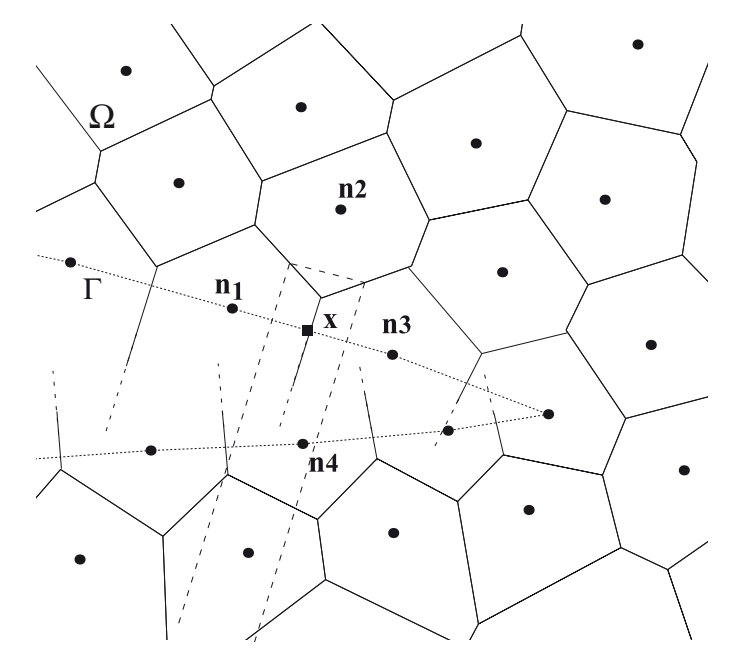

Figure 6. Computation of natural neighbor shape functions over non convex domain by mean of the constrained Voronoi diagram

With this technique, it is trivial to introduce holes, inclusions or discontinuities by simply adding boundary edges (in 2D) or facets (in 3D) in the boundary description. In (Yvonnet et al., 2004), we have shown that the properties of partition of unity and linear consistency were met in the crack-tip field neighbourhood with the C-NEM technique. 
In order to demonstrate the potential of the C-NEM approach for crack analysis, a numerical example is provided. We examine the case of a centrally located inclined crack of length $2 \mathrm{a}=0.2$ units and an inclination $\gamma$ in a finite two dimensional square plate of size $2 \mathrm{~W} \times 2 \mathrm{~W}=2$ units, as shown in Figure 7. Plane stress conditions were assumed with elastic modulus $E=1 \mathrm{MPa}$ and Poisson's ratio $\nu=0.3$. A constant load of $\sigma_{22}^{\infty}$ on both lower and upper sides of the square is applied. We first consider the case where $\gamma=0$.

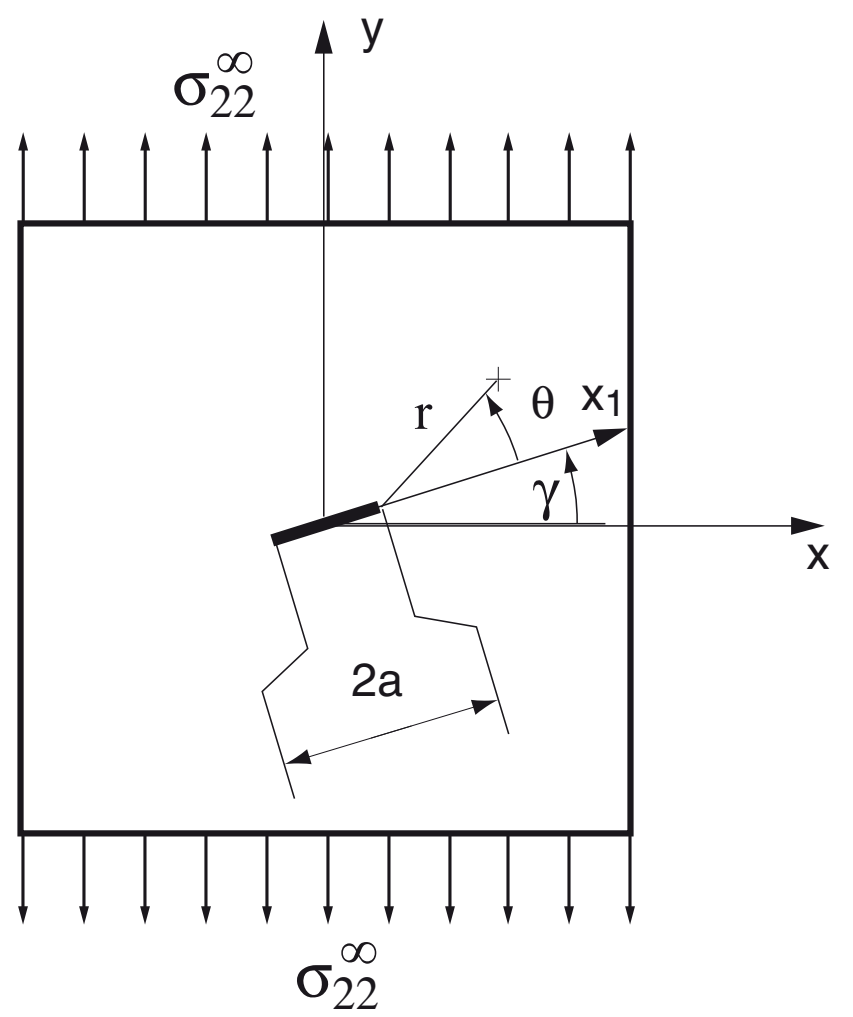

Figure 7. Modelisation of the plate with an interior inclined crack

This problem has an analytical solution, called Muskelishvili's solution. This solution involves an infinite plate that can not be represented by meshless discretisation. Nevertheless, if the dimension of the crack is small compared with the size of the domain, the assumption is reasonable. In this example, we took $\mathbf{W}=10$ a. For $\gamma=0$, $\theta=0$, the exact solution is given by :

$$
\begin{aligned}
& \sigma_{22}(\theta=0, \gamma=0)=\sigma_{22}^{\infty} \frac{a+r}{\sqrt{r(2 a+r)}} \\
& \sigma_{11}(\theta=0, \gamma=0)=\sigma_{22}^{\infty}-\sigma_{22}
\end{aligned}
$$

No symmetry is considered in the nodal distribution. Numerical results are depicted in Figure 8. Now, we consider the case of an inclined crack. The stress intensity factor (SIF) $K_{I}$ is computed using the interaction integral method, and compared in Figure 9 with the analytical solution (Lemaitre and Chaboche, 1990):

$$
K_{I}=\sigma_{22}^{\infty} \sqrt{\pi a} \cos ^{2} \gamma
$$




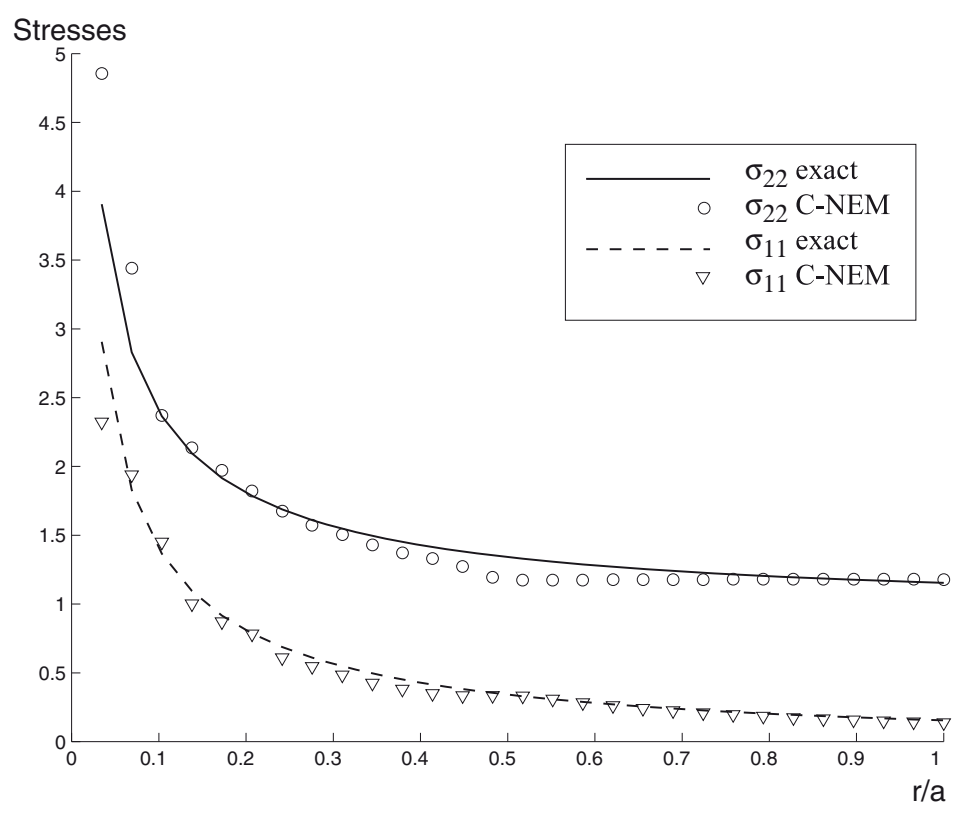

Figure 8. Radial stresses ahead of the crack tip for $\gamma=0$

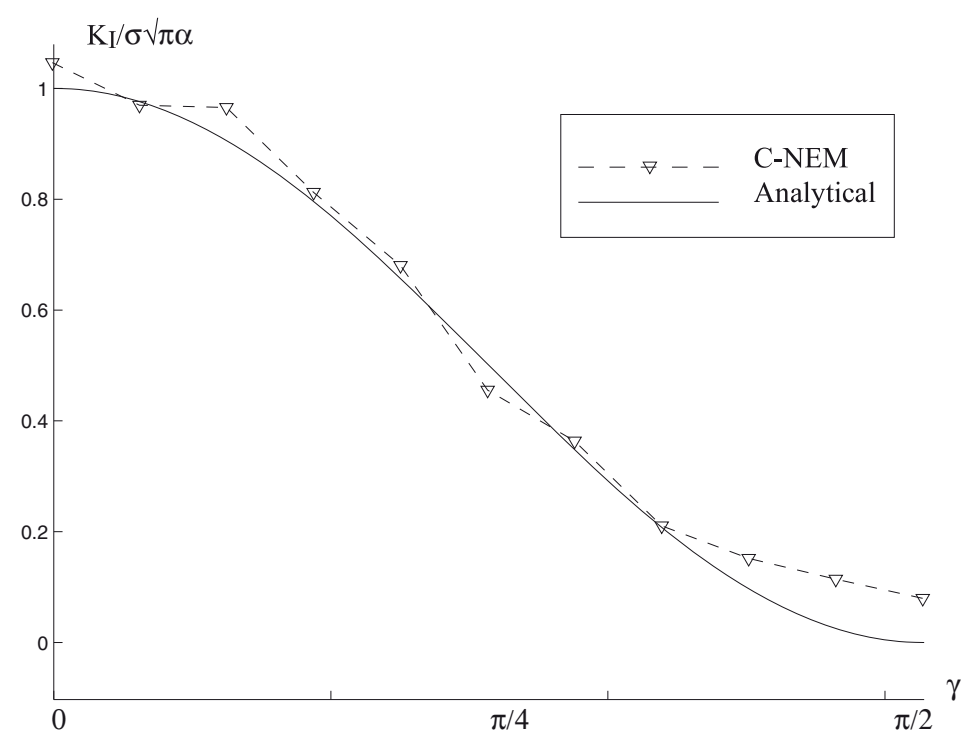

Figure 9. Normalized stress intensity factor $K_{I}$ for different values of $\gamma$

Distance between the nodes near the crack tip is about $0.1 \mathrm{a}$. It can be noticed from Figs. 8 and 9 that despite of this coarse nodal discretisation, C-NEM solution is in reasonable agreement with the analytical solution. Accuracy can be improved by adding nodes in the tip neighbourhood or by enriching shape functions in the framework of the partition of unity method (Babuška and Melenk, 1996).

\section{An Enriched Assumed Strain Method}

Recently, much attention has been focused on a class of finite element methods that allow for the representation of strong intra-element discontinuities. Among the more notable are the enhanced assumed strain approaches (Armero and Garikipati, 1996) 
(Steinmann et al., 1997), wherein the discontinuous mode is introduced through an enhanced deformation gradient approximation. In what follows, we provide a summary of a new formulation presented in (Dolbow and Devan, 2003) that incorporates the intra-element discontinuities directly at the approximation level using an enrichment strategy based upon the partition-of-unity framework (Melenk and Babuška, 1996). The new method retains the enhancement to the assumed strain field, as it provides for coarse-mesh accuracy as well as a means to address volumetric incompressibility constraints. We propose a modification to the enhanced strain basis that allows it to be orthogonal to a piecewise-constant stress field in "cut" elements. The goal is to obtain a robust method for high-speed machining, for example, where large deformations and the isochoric nature of plastic flow place severe demands on the simulation technology.

We consider the body identified with a bounded region $\mathcal{R}$ of three-dimensional Euclidean point space $\Re^{3}$ that it occupies in a fixed reference configuration. We label particles in this reference configuration by $\boldsymbol{X} \in \mathcal{R}$ with coordinates $\boldsymbol{X}=$ $\left(X_{1}, \ldots, X_{n_{\text {dim }}}\right)$. The deformation $\varphi$ is assumed to satisfy Dirichlet boundary conditions $\varphi=\hat{\varphi}$ on $\partial_{u} \mathcal{R} \subset \partial \mathcal{R}$. Since we neglect inertial forces, the deformational force system consists of the bulk stress $\boldsymbol{P}$, and imposed tractions $\overline{\boldsymbol{T}}$ on $\partial_{t} \mathcal{R} \subset \partial \mathcal{R}$. We allow bulk fields to be discontinuous across the smooth material surface $\mathcal{S}$. In the present study, we will assume the surface $\mathcal{S}$ to be traction-free.

The variational formulation we employ follows from a three-field $\mathrm{Hu}$-Washizu functional in $\{\boldsymbol{\varphi}, \boldsymbol{P}, \tilde{\boldsymbol{F}}\}$. Here, $\tilde{\boldsymbol{F}}$ denotes an additional enhanced field to the deformation gradient. Precisely, the deformation gradient takes the assumed form

$$
\boldsymbol{F}=\operatorname{GRAD} \varphi+\tilde{\boldsymbol{F}}
$$

where GRAD is the gradient operator in the reference configuration.

We let $\mathcal{V}$ denote the space of sufficiently regular motions, $\mathcal{L}$ the space of sufficiently regular stresses and enhanced deformation gradients. The variational boundary value problem is then : Find $(\boldsymbol{\varphi}, \boldsymbol{P}, \tilde{\boldsymbol{F}}) \in \mathcal{V} \times \mathcal{L} \times \mathcal{L}$ such that

$$
\begin{aligned}
& \int_{\mathcal{R}} 2 \boldsymbol{F} \frac{\partial \hat{W}(\boldsymbol{C})}{\partial \boldsymbol{C}} \cdot \operatorname{GRAD} \delta \boldsymbol{\varphi} d V=\int_{\partial_{t} \mathcal{R}} \overline{\boldsymbol{T}} \cdot \delta \boldsymbol{\varphi} d V, \\
& \int_{\mathcal{R}}\left(2 \boldsymbol{F} \frac{\partial \hat{W}(\boldsymbol{C})}{\partial \boldsymbol{C}}-\boldsymbol{P}\right) \cdot \delta \tilde{\boldsymbol{F}} d V=0, \\
& \int_{\mathcal{R}} \tilde{\boldsymbol{F}} \cdot \delta \boldsymbol{P} d V=0,
\end{aligned}
$$

for all arbitrary variations $(\delta \boldsymbol{\varphi}, \delta \boldsymbol{P}, \delta \tilde{\boldsymbol{F}}) \in \mathcal{V} \times \mathcal{L} \times \mathcal{L}$. In the above equations, $\hat{W}$ is the bulk strain-energy density function and $C$ is the right Cauchy-Green tensor, $\boldsymbol{C}=\boldsymbol{F}^{T} \boldsymbol{F}$. 
For the sake of concreteness, we consider a standard finite element mesh of fournode quadrilateral elements with nodal shape functions $\phi_{i}$. The approximation to the deformation field is written as

$$
\boldsymbol{\varphi}^{h}(\boldsymbol{X})=\sum_{i \in I} \boldsymbol{a}_{i} \phi_{i}(\boldsymbol{X})+\sum_{j \in J} \boldsymbol{b}_{j} \phi_{j}(\boldsymbol{X}) H_{d}(\boldsymbol{X}),
$$

where $I$ denotes the set of all nodes in the mesh and $J$ the subset that are enriched with the Heaviside function $H_{d}$. The vectors $\boldsymbol{a}_{i}$ and $\boldsymbol{b}_{j}$ are constant degrees of freedom.

Considering a closed subset $\Omega \subset \mathcal{R}$ that is partitioned into complementary subregions $\omega^{+}$and $\omega^{-}$by the discontinuity $\mathcal{S}$, the Heaviside function is given by

$$
H_{d}(\boldsymbol{X})= \begin{cases}1 & \text { for } \boldsymbol{X} \in \omega^{+} \\ 0 & \text { for } \boldsymbol{X} \in \omega^{-}\end{cases}
$$

The set $J$ of nodes enriched with this function is determined from the "interaction" between the set of overlapping subdomains $\left\{\omega_{i}\right\}$ defining the support of each nodal shape function and the geometry $\mathcal{S}$ (Moës et al., 1999) (Dolbow et al., 2000). Since we do not incorporate asymptotic near-tip functions into the approximation, the present work is slightly restricted by the need for the discontinuity to terminate on an element boundary.

On each element $\mathcal{R}_{e}$, we consider approximations for $\boldsymbol{F}^{h}$ of the form

$$
\boldsymbol{F}^{h}(\boldsymbol{\xi})=\left.\operatorname{GRAD} \boldsymbol{\varphi}^{h}\right|_{\mathcal{R}_{e}}+\sum_{i \in E} \boldsymbol{\alpha}_{i} \otimes \boldsymbol{G}_{i}(\boldsymbol{\xi})
$$

where $E$ denotes the set of enhancement functions on the element. In the above, $\boldsymbol{G}_{i}$ denotes the vector-valued local enhancement functions written in terms of the parent coordinates $\boldsymbol{\xi}$, with $\boldsymbol{\alpha}_{i}$ the corresponding degrees of freedom.

In the spirit of earlier efforts (Simo and Armero, 1992), we construct functions $G_{i}$ that are orthogonal to a subset of the fields spanned by the gradient of [22]. In particular, for those elements intersected by $\mathcal{S}$, we seek to construct vector-valued functions $\mathrm{G}_{i}$ that satisfy

$$
\int_{\square^{+}} \mathbf{G}_{i}(\boldsymbol{\xi}) d \square=\mathbf{0}, \quad \int_{\square^{-}} \mathbf{G}_{i}(\boldsymbol{\xi}) d \square=\mathbf{0} .
$$

where $\square^{+}$and $\square^{-}$denote the complementary subsets of the parent domain formed by the intersection of $\square$ and the image of $\mathcal{S}$. These functions are then mapped to the reference domain with a constant Jacobian matrix (Simo and Armero, 1992). As such, the functions $\boldsymbol{G}_{i}$ are orthogonal to piecewise-constant stress fields in "cut" elements, enabling the formulation to satisfy a discontinuous version of the patch test (Dolbow and Devan, 2003). This is in marked contrast to the work of (Armero and Garikipati, 1996), where the discontinuous mode was constructed to be orthogonal to a constant stress field only. 
We begin with functions $G_{i}$ that are shifted and linear in $\boldsymbol{\xi}$. For example, in $\square^{+}$, we use

$$
\mathbf{G}_{1}(\boldsymbol{\xi})=\left[\begin{array}{c}
\xi_{1}-\bar{\xi}_{1}^{+} \\
0
\end{array}\right], \quad \mathbf{G}_{2}(\boldsymbol{\xi})=\left[\begin{array}{c}
0 \\
\xi_{2}-\bar{\xi}_{2}^{+}
\end{array}\right], \mathrm{G}_{3}(\boldsymbol{\xi})=\mathrm{G}_{4}(\boldsymbol{\xi})=0
$$

The shift points, $\bar{\xi}^{+}$in $\square^{+}$and $\bar{\xi}^{-}$in $\square^{-}$, are determined such that [25] is satisfied during numerical integration. An analogous construction is employed on $\square^{-}$, with $\mathrm{G}_{3}$ and $\mathrm{G}_{4}$ non-zero. For elements not intersected by $\mathcal{S}$, only the first two functions above are employed and the formulation reverts to that proposed in (Simo and Armero, 1992).

Substituting the approximations for the deformation and the enhanced gradient into the Galerkin form of [21] gives rise to a nonlinear system of algebraic equations. This system is resolved using Newton-Raphson iteration to obtain the degrees of freedom $\boldsymbol{a}_{i}, \boldsymbol{b}_{j}$, and $\boldsymbol{\alpha}_{i}$. The process is identical to that described in (Simo and Armero, 1992), with the exception of the modified integration schemes employed in the enriched elements.

\section{A fractured Cook's membrane problem}

We consider finite deformation elastic problems wherein the response is governed by a nearly incompressible neo-Hookean material model. In particular, we employ a stored energy function of the form

$$
\hat{W}=\frac{\mu}{2}\left[J^{-2 / 3} \operatorname{tr}[\boldsymbol{C}]-3\right]+\frac{\kappa}{2}(J-1)^{2},
$$

with shear modulus $\mu$ and bulk modulus $\kappa$. In order to examine the nearly incompressible limit, we select $\kappa / \mu \approx 10^{4}$.

We consider a tapered, fractured panel clamped on one end and subjected to a shearing load on the other as shown in Figure 10. Without the discontinuity, this problem is often referred to as 'Cook's membrane problem'.

To evaluate the accuracy of the modified enriched/enhanced formulation for the problem with a discontinuity, we compare our results to those obtained when the crack is explicitly meshed. The enriched and explicitly meshed discretizations are shown in Figures $2 \mathrm{a}$ and $2 \mathrm{~b}$, respectively. When the crack is meshed, no modification to the standard assumed strain functions are necessary as the discontinuity is represented by the doubled nodes along $\mathcal{S}$.

We perform calculations using 30 load steps with increments of $\Delta F=1.0 \mathrm{~N}$. In all calculations, we observe an asymptotic quadratic rate of convergence in the Euclidean norm of the residual. The deformed meshes are compared in Figure 10c, and we note the large deformation and close match of the results. Both approximations satisfy the volumetric constraint reasonably well, with the maximum in $|\operatorname{det} \boldsymbol{F}-1|$ typically occurring near the crack tip, but less than 5\%. We conclude that the modified assumed strain method does not exhibit volumetric locking, and furthermore provides a means 


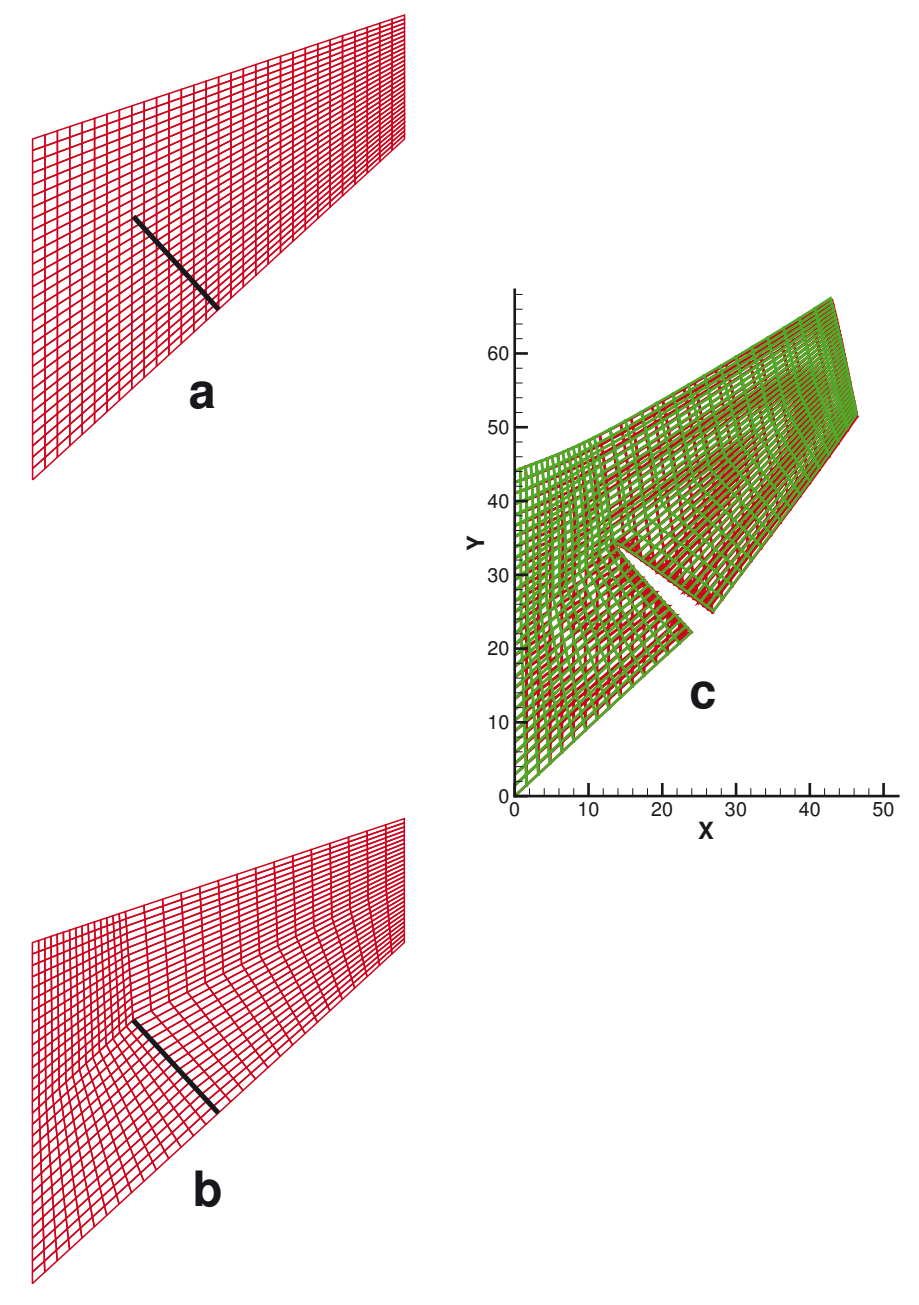

Figure 10. Meshes employed for the (a) enriched and (b) standard approximations.The heavy solid line denotes the crack location. (c) Overlay of the final deformed meshes without magnification. All results shown are for the nearly incompressible case, using $\kappa / \mu=10^{4}$

to represent an evolving discontinuity without any remeshing. Finally, we note that the assumed strain framework also lends this method coarse-mesh accuracy.

\section{Conclusions}

We reviewed MLS- and natural neighbor-based meshless methods. The positive attributes in the latter were the ease of imposing essential boundary conditions, and the construction of robust approximations at a relatively low cost. It is simpler to construct $C^{k}$ trial spaces using MLS approximants. Errors due to numerical integration in meshless methods demand attention. Rigorous mathematical analysis of meshless methods is required to develop a better understanding of these methods, and to realize their full potential. Partition of unity methods are clearly superior when discontinuous phenomena, singularities, or small-scale features need to be captured on a coarse mesh. The partition of unity framework is particularly advantageous for $2-\mathrm{d}$ and $3-\mathrm{d}$ 
crack growth simulations; meshless methods such as EFG have had limited success in 3-d crack modeling (Sukumar et al., 1997).

\section{Acknowledgements}

The support of the National Science Foundation grant number DMI-0223611, to Duke University, is gratefully acknowledged. Also, the support of the Spanish Ministry of Science and Technology, through the project CICYT-DPI2002-01986 is gratefully acknowledged.

\section{References}

Armero F. and Garikipati K. "An analysis of strong discontinuities in multiplicative finite strain plasticity and their relation with the numerical simulation of strain localization in solids". International Journal of Solids and Structures, vol. 33, n 20-22, 1996, p. 2863-2885.

Babuška I. and Melenk J. M. “The partition of unity finite element method: Basic theory and applications". Comp. Meth. in Appl. Mech. and Eng. Vol. 4, 1996, p. 289-314.

Belytschko T., Krongauz Y., Organ D., Fleming M. and Krysl P. "Meshless methods: An overview and recent developments". Computer Methods in Applied Mechanics and Engineering. Vol. 139, 1998, p. 3-47.

Braun J. and Sambridge M. "A numerical method for solving partial differential equations on highly irregular evolving grids". Nature. Vol. 376, 1995, p. 655-660.

Christ N. H., Friedberg R. and Lee T. D. "Weights of links and plaquettes in a random lattice". Nuclear Physics B. Vol. 210, nํㅡㄹ 1982, p. 337-346.

Cueto E., Calvo B. and Doblaré M. "Modeling three-dimensional piece-wise homogeneous domains using the $\alpha$-shape based Natural Element Method". International Journal for Numerical Methods in Engineering. Vol. 54, 2002, p. 871-897.

Cueto E., Cegoñino J., Calvo B. and Doblaré M. "On the imposition of essential boundary conditions in Natural Neighbour Galerkin methods". Communications in Numerical Methods in Engineering. Vol. 19(5), 2003, p. 361-376.

Cueto E., Doblaré M. and Gracia L. "Imposing essential boundary conditions in the Natural Element Method by means of density-scaled $\alpha$-shapes". International Journal for Numerical Methods in Engineering. Vol. 49-4, 2000, p. 519-546.

Cueto E., Sukumar N., Calvo B., Cegoñino J. and Doblaré M. "Overview and recent advances in Natural Neighbour Galerkin methods". Archives of Computational Methods in Engineering. Vol. 10(4), 2003, p. 307-384.

Dolbow J. An extended finite element method with discontinuous enrichment for applied mechanics. $\mathrm{Ph}$. D. Thesis Northwestern University (1999).

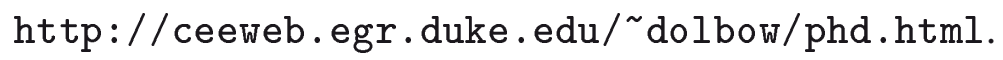


Dolbow J., Moës N. and Belytschko T. "Discontinuous enrichment in finite elements with a partition of unity method". Finite Elements in analysis and design. Vol. 36, 2000, p. 235260.

Dolbow J. E. and Devan J. E. "Enrichment of enhanced assumed strain methods for representing strong discontinuities". International Journal for Numerical Methods in Engineering. In press. Preprint available at http://ceeweb.egr.duke.edu/ dolbow/strong.html. 2003.

Duarte C. A. M. and Oden J. T. "An H-p Adaptive Method using Clouds". Computer Methods in Applied Mechanics and Engineering. Vol. 139, 1996, p. 237-262.

Edelsbrunner H., Kirkpatrick D. G. and Seidel R. "On the shape of a set of points in the plane". IEEE Transactions on Information Theory. Vol. IT-29(4), 1983, p. 551-559.

Edelsbrunner H. and Mücke E. "Three dimensional alpha shapes". ACM Transactions on Graphics. Vol. 13, 1994, p. 43-72.

Lancaster P. and Salkauskas K. "Surfaces generated by moving least squares methods". Mathematics of Computation. Vol. 37, 1981, p. 141-158.

Lemaitre J. and Chaboche J.-L. Mechanics of solid materials. Cambridge University Press, 1990.

Li S. and Liu W. K. "Meshfree and particle methods and their applications". Applied Mechanics Review. Vol. 55, n- 1,2002 , p. 1-34.

Melenk J. M. and Babuška I. "The partition of unity finite element method: Basic theory and applications". Computer Methods in Applied Mechanics and Engineering. Vol. 139, 1996, p. 289-314.

Moës N., Dolbow J. and Belytschko T. "A finite element method for crack growth without remeshing". International Journal for Numerical Methods in Engineering. Vol. 46, 1999, p. 131-150.

Nayroles B., Touzot G. and Villon P. "Generalizing the finite element method: Diffuse approximation and diffuse elements”. Computational Mechanics. Vol. 10, 1992, p. 307-318.

Organ D., Fleming M., Terry T. and Belytschko T. "Continuous meshless approximations for nonconvex bodies by difraction and transparency". Computational Mechanics. Vol. 18, 1996, p. 1-11.

Sethian J. A. "Fast marching methods". SIAM Review. Vol. 41, nํ 2, 1999, p. 199-235.

Sibson R. "A Vector Identity for the Dirichlet Tesselation". Mathematical Proceedings of the Cambridge Philosophical Society Vol. 87, 1980, p. 151-155.

Sibson R. "A brief description of natural neighbour interpolation". In Interpreting Multivariate Data. V. Barnett (Editor) 21-36. John Wiley (1981).

Simo J. C. and Armero F. "Geometrically non-linear enhanced strain mixed methods and the metod of incompatible modes". International Journal Numerical methods in Engineering. Vol. 46, 1992, p. 131-150. 
Steinmann P., Larsson R. and Runessson K. "On the localization properties of multiplicative hyperelasto-plastic continua with strong discontinuities". International Journal of Solids and Structures. Vol. 34(8), 1997, p. 969-990.

Sukumar N. The Natural Element Method in Solid Mechanics. Ph. D. Thesis Northwestern University Evanston, Illinois (1998).

Sukumar N., Chopp D. L., Moës N. and Belytschko T. "Modeling Holes and Inclusions by Level Sets in the Extended Finite Element Method". Computer Methods in Applied Mechanics and Engineering. Vol. 190(46-47), 2001, p. 6183-6200.

Sukumar N., Moran B. and Belytschko T. "The Natural Element Method in Solid Mechanics". International Journal for Numerical Methods in Engineering. Vol. 43(5), 1998, p. 839887.

Sukumar N., Moran B., Black T. and Belytschko T. "An element-free Galerkin method for threedimensional fracture mechanics". Computational Mechanics. Vol. 20, 1997, p. 170-175.

Wagner G. J. and Liu W. K. "Hierarchical enrichment for bridging scales and mesh-free boundary conditions". International Journal for Numerical Methods in Engineering. Vol. 50, n- 3,2001, p. 507-524.

Yvonnet J., Ryckelynck D., Lorong P. and Chinesta F. "A new extension of the Natural Element method for non-convex and discontnuous problems: the Constrained Natural Element method". International Journal for Numerical Methods in Enginering. Vol. 60, 2004, p. 1451-1474. 